DRAFT VERSION 2018 OCTOBER 14

Preprint typeset using $\mathrm{LTT}_{\mathrm{E} X} \mathrm{X}$ style emulateapj v. 5/2/11

\title{
ON THE DENSITY PROFILE OF THE GLOBULAR CLUSTER M92 ${ }^{1}$
}

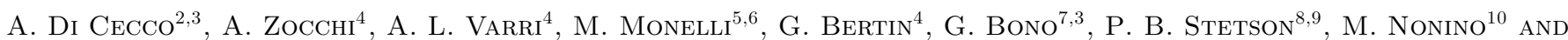 \\ R. Buonanno ${ }^{7,2}$, I. Ferraro ${ }^{3}$, G. Iannicola ${ }^{3}$, A. Kunder ${ }^{11}$, A. R. Walker ${ }^{11}$
}

(Dated: drafted 2018 October 14 / Received / Accepted)

Draft version 2018 October 14

\begin{abstract}
We present new number density and surface brightness profiles for the globular cluster M92 (NGC 6341). These profiles are calculated from optical images collected with the CCD mosaic camera MegaCam at the Canada-France-Hawaii-Telescope and with the Advanced Camera for Surveys on the Hubble Space Telescope. The ground-based data were supplemented with the Sloan Digital Sky Survey photometric catalog. Special care was taken to discriminate candidate cluster stars from field stars and to subtract the background contamination from both profiles. By examining the contour levels of the number density, we found that the stellar distribution becomes clumpy at radial distances larger than $\sim 13^{\prime}$, and there is no preferred orientation of contours in space. We performed detailed fits of King and Wilson models to the observed profiles. The best-fit models underestimate the number density inside the core radius. Wilson models better represent the observations, in particular in the outermost cluster regions: the good global agreement of these models with the observations suggests that there is no need to introduce an extra-tidal halo to explain the radial distribution of stars at large radial distances. The best-fit models for the number density and the surface brightness profiles are different, even though they are based on the same observations. Additional tests support the evidence that this fact reflects the difference in the radial distribution of the stellar tracers that determine the observed profiles (main sequence stars for the number density, bright evolved stars for the surface brightness).

Subject headings: globular clusters: general — globular clusters: individual (M92) — stars: kinematics and dynamics — stars: Population II
\end{abstract}

\section{INTRODUCTION}

The Galactic globular clusters (GCs) are the oldest ( 11-13 Gyr) Galactic systems and have complex internal and external dynamics (Gnedin \& Ostriker 1997). The internal dynamics is driven by two-body relaxation with a time scale that is typically shorter than their age (Meylan \& Heggie 1997). Therefore, the density profile of the innermost regions is expected to be well described

\footnotetext{
${ }^{1}$ Based in part on data obtained from the ST-ECF Science Archive Facility. This research used the facilities of the Canadian Astronomy Data Centre operated by the National Research Council of Canada with the support of the Canadian Space Agency.

2 Agenzia Spaziale Italiana Science Data Center (ASDC), c/o ESRIN, via G. Galilei, 00044 Frascati, Italy

${ }^{3}$ Istituto Nazionale di Astrofisica (INAF) - Osservatorio Astronomico di Roma, Via Frascati 33, 00044 Monte Porzio Catone, Italy

4 Università degli Studi di Milano, Dipartimento di Fisica, via Celoria 16, 20133 Milano, Italy

${ }^{5}$ Instituto de Astrofisica de Canarias, Calle Via Lactea, E38200 La Laguna, Tenerife, Spain

${ }^{6}$ Departamento de Astrofisica, Universidad de La Laguna, Tenerife, Spain

7 Dipartimento di Fisica, Università di Roma Tor Vergata, via della Ricerca Scientifica 1, 00133 Rome, Italy

8 Dominion Astrophysical Observatory, Herzberg Institute of Astrophysics, National Research Council, 5071 West Saanich Road, Victoria, BC V9E 2E7, Canada

${ }^{9}$ Visiting Astronomer, Cerro Tololo Inter-American Observatory, National Optical Astronomy Observatories, operated by AURA, Inc., under cooperative agreement with the NSF.

${ }_{10}$ INAF-Osservatorio Astronomico di Trieste, via G.B. Tiepolo 11, 40131 Trieste, Italy

${ }^{11}$ Cerro Tololo Inter-American Observatory, National Optical Astronomy Observatory, Casilla 603, La Serena, Chile
}

by King models $(1962,1966)$. The outermost regions are characterized by the interaction with external tidal forces (Spitzer 1958; Spitzer \& Chavalier 1973; Aguilar et al. 1988) and by the evaporation of low-mass stars (Spitzer \& Harm 1958). These phenomena are expected to produce deviations from the spherical King models. By moving beyond the cluster truncation radius $\left(r_{t}\right)$ the escaping stars can form halos or extended tidal tails.

The first empirical evidence of extra-tidal structures in GCs was found from photographic plates (Grillmair et al. 1995; Lehmann \& Scholz 1997; Testa et al. 2000 [T00]; Leon et al. 2000). More recently, investigations based on CCD photometry found evidence either of tidal tails (Odenkirchen et al. 2001,2003; Grillmair \& Johnson 2006; Chun et al. 2010; Jordi \& Grebel 2010 [JG10]) or of surrounding halos (Lee et al. 2003 [L03]; Olszewski et al. 2009; JG10; Correnti et al. 2011) around more than 30 GCs.

An accurate determination of the truncation radius of M92 and of the shape of its external regions is still missing, even though several investigations have been carried out on this topic (see Table 1). The oldest Surface Brightness (SB) profile (obtained from photographic plates and small-format CCD images) was provided by Trager et al. (1995, [T95]). The same was later analyzed by McLaughlin \& van der Marel (2005, [MLvdM05]). By using photographic plates, T00 found evidence of extratidal stars at $30^{\prime}$ from the cluster center, and provided a surface density map, which shows only marginal evidence for an elongation orthogonal to the direction of the Galactic center. More recently, L03 by using a mosaic CCD camera, confirmed the occurrence of extra- 
tidal stars and showed that the marginal elongation appears only for the brightest stars. JG10 analyzed the Sloan Digital Sky Survey (SDSS) photometric catalog and found the same elongated contours detected by T00, even though their data do not cover the entire M92 area.

\section{PHOTOMETRIC DATASETS}

We used both ground-based data collected with the 36 CCD mosaic camera MegaCam at the Canada-FranceHawaii-Telescope (CFHT) and space data collected with the Advanced Camera for Surveys (ACS) on the Hubble Space Telescope (HST). The MegaCam images were collected in the $g^{\prime}, r^{\prime}, i^{\prime}, z^{\prime}$ band $\$^{12}$. To increase the radial extent of the sky area covered by our dataset, the groundbased data (MegaCam images) were supplemented with multiband $(g, r, i, z)$ data collected by the SDSS (Aihara et al. 2011). As a whole the ground-based data cover $\sim 4^{\circ} \times 4^{\circ}$ around the cluster center, but they do not uniformly cover the sky area around M92.

The ACS data were collected with three different pointings: pointing $\alpha$, High Resolution Channel (HRC), six $\mathrm{F} 435 \mathrm{~W}\left(\mathrm{t}=340 \mathrm{~s}\right.$ each) and $155 \mathrm{~F} 555 \mathrm{~W}^{13}$ (with exposure times ranging from 10 to $100 \mathrm{~s}$ ) images located across the cluster center; pointing $\beta$, Wide Field Channel (WFC), three F606W ( $\mathrm{t}=0.5,5,90 \mathrm{~s})$ and three $\mathrm{F} 814 \mathrm{~W}^{\mathrm{T4}}$ $(\mathrm{t}=0.5,6,100 \mathrm{~s})$ images located across the cluster center; pointing $\gamma$, WFC, three $\mathrm{F} 475 \mathrm{~W}(\mathrm{t}=3,20,40 \mathrm{~s})$ and three F814W $15(t=1,10,20 \mathrm{~s})$ images located $2^{\prime}$ from the cluster center in the South-East direction. The reduction and the photometry of the F555W and F814W images have been presented and discussed by Di Cecco et al. (2010, [DiC10]). Panels a) and b) of Fig. 1 display the sky coverage of the space- and ground-based datasets.

To estimate the completeness of the CFHT data we adopted the data of the pointing $\gamma$ (ACS-WFC). We found that the completeness for $i \leq 22 \mathrm{mag}$ is $54 \%$ for $75 \leq \mathrm{R} \leq 150$ arcsec, $82 \%$ for $150 \leq \mathrm{R} \leq 200$ arcsec, and complete for larger distances. We applied the above completeness corrections to the star counts (DiC10). We also estimated the completeness of the SDSS data by using the CFHT data and we found that for radial distances larger than 700 arcsec and $i \leq 22$ mag they are complete. For radial distances smaller than $75^{\prime \prime}$ we adopted the data of pointing $\beta$. These data display a gap of $2.5^{\prime \prime}$ between the two CCDs. To fill this gap we selected two regions of $2.5^{\prime \prime}$ at the edges of the gap and we randomly extracted half of the stars in each of the two regions. Once the gap was filled, we assumed that the data of pointing $\beta$ located between $20^{\prime \prime}$ and $75^{\prime \prime}$ are complete (DiC10). The completeness of pointing $\beta$, for radial distance $-R$ - smaller than $11^{\prime \prime}$, was estimated using pointing $\alpha$. In this case we found that in the magnitude range between the Main Sequence Turn Off (MSTO) and F555W 21.5 mag the pointing $\beta$ dataset was complete at the $70 \%$ level. The star counts in this cluster region were corrected accounting for the above completeness correction.

In summary, we are dealing with three different datasets:

1) $-\mathrm{ACS}-\mathrm{WFC}$, pointing $\beta, R \leq 1.25^{\prime}$. For radial

12 Proposal ID: 2004AC03, PI: J. Clem

13 GO-10335, PI: H. Ford

14 GO-9453, PI: T. Brown

15 GO-10505, PI: C. Gallart distances smaller than $0.18^{\prime}$ the completeness was estimated using ACS-HRC, pointing $\alpha$. For radial distances $0.18^{\prime} \leq R \leq 1.25^{\prime}$ we only filled the gaps.

2)- CFHT, $1.25^{\prime}<R \leq 30^{\prime}$. For radial distances $1.25^{\prime}<R \leq 3.33^{\prime}$ the completeness was estimated using ACS-WFC, pointing $\gamma$. The comparison of CFHT with pointing $\gamma$ indicates that the former dataset for $i \leq 22$ mag is complete at larger distances.

3)- SDSS, $30^{\prime}<R \leq 2^{\circ}$. The completeness was estimated using CFHT data; they are complete for $i \leq 22$ mag and radial distances larger than 700 arcsec. However, they do not uniformly cover the sky region around the cluster (see panel b) in Fig. 1) and the star counts were accordingly corrected.

Panels a), b) and c) of Fig. 2 show the color-magnitude diagrams (CMDs) of pointing $\alpha, \beta$ and $\gamma$. Stars plotted in these panels were selected according to the sharpness $(|\operatorname{sh}| \leq 1)$ DAOPHOT index (see, e.g., Stetson 1987, 1994). These CMDs display well defined sequences in the evolved phases as well as along the Main Sequence (MS). The Red Giant Branch (RGB) stars brighter than red Horizontal Branch (HB) stars (F555W 14 mag) are saturated in pointing $\alpha$.

To provide homogeneous star counts across the entire GC, the $i$ - and the $r$-band from the SDSS, as well as the $F 814 W$ - and the $F 606 W$-band of pointing $\beta$ were transformed into the $i$ '- and the $r^{\prime}$-band of the MegaCam photometric system. The accuracy of the transformations is better than $0.02 \mathrm{mag}$ ( $\mathrm{DiC} 10)$. In the following, we use the $g, r, i, z$ bands (without prime) to refer to the CFHT bands. The reason for the above transformations is threefold: $a$ ) $i$ - and $r$-band are common to the different datasets; $b$ ) the data in these bands have good photometric accuracy $\left(\sigma_{\mathrm{r}-\mathrm{i}}=0.06 \mathrm{mag}\right.$, at least three magnitudes fainter than the MSTO); c) the $i$-band is minimally affected by saturation problems and it was adopted to compute the density profiles.

\section{RADIAL DENSITY PROFILE}

The field of view of the CFHT dataset $\left(1^{\circ} \times 1^{\circ}\right)$ and of the SDSS dataset fully enclose the estimated radial extent of M92 (see Table 1). In order to constrain the cluster edges, candidate cluster and field stars must be distinguished. The method used to identify the two different groups of star is described below.

We selected the photometric catalog by using the intrinsic photometric error $\left(\sigma_{\mathrm{r}-\mathrm{i}} \leq 0.10 \mathrm{mag}\right)$, the separation 16 ( $s e p \geq 2.5)$, and the distance from the cluster center $\left(10^{\prime \prime} \leq R \leq 180^{\prime \prime}\right)$. We computed a fiducial line (ridgeline) in the $i, r-i$ CMD by means of a three-dimensional Hess diagram (Ferraro et al. 2013, in preparation). Note that the above selection criteria were only applied to estimate the ridge line.

Panel a) of Fig. 3 shows the $i, r-i$ CMD for the entire sample of stars together with the above ridgeline. Stars plotted in this figure were selected according to the radial distance and to a very mild photometric error cut $\left(\sigma_{\mathrm{r}-\mathrm{i}} \leq 0.25 \mathrm{mag}\right)$. Note that in this analysis we only adopted space and CFHT data; the SDSS data will be discussed in the following. The solid line shows

\footnotetext{
16 The separation index quantifies the degree of crowding, i.e. the amount of spurious light, due to neighboring stars, affecting the magnitude of individual stars (Stetson et al. 2003).
} 
the computed ridgeline, whereas the dashed ones display the acceptance region, that is, the region of the CMD where we assume that the candidate MS and RGB cluster stars lie. The acceptance region is centered on the ridgeline and the width in color goes from 0.01 mag close to the tip of the RGB up to 0.30 mag for magnitudes fainter than the MSTO. We cut the acceptance region at magnitudes brighter than $i=21.7 \mathrm{mag}$, because at fainter magnitudes the photometric error in the color increases and the ridgeline is less well determined.

Panel b) of Fig. 3 shows the accepted M92 stars, that is, the candidate MS, RGB, and HB stars. The MS and RGB accepted cluster stars include some candidate field stars with colors and magnitudes similar to those of M92. The HB stars, instead, can be easily selected since they are bluer than the field stars. Panel c) of Fig. 3 shows the CMD of candidate field stars (rejected) with their typical peaks in color around $r-i=0-0.2$ and $r-i=1.2-1.4$ mag. The stars located at $(i, r-i) \sim(17.5,0.0)$ are M92 Blue Stragglers. They were not included among accepted stars, because the fainter ones partially overlap with field and MSTO stars in the CMD, while the brighter ones are a minimal fraction of cluster stars.

To further constrain the radial extent of candidate cluster stars we investigated the ratio between the number of accepted stars and the total number of stars in radial bins located in the outermost cluster regions. Data plotted in Fig. 4 show the $i, r-i$ CMD for stars in radial bins located between $13^{\prime}$ and $2^{\circ}$ from the cluster center. The first three radial bins -panel a), b) and c- are entirely included inside the CFHT dataset, while the last two -panel d) and e)- are entirely located inside the SDSS dataset. The two solid lines display the acceptance region we defined in Fig. 3. The photometric precision in the outermost cluster regions is clearly supported by the thin distribution of MS stars. Data plotted in the three innermost radial bins indicate that MS stars are crucial to trace the radial extent of candidate cluster stars. Moreover, the ratio between the number of cluster stars and the total number of stars is steadily decreasing when moving toward the outermost cluster regions. It decreases from almost $50 \%$ $\left(N_{A} / N_{T}=0.49 \pm 0.02\right)$ for $\mathrm{R} \sim 14.5^{\prime}$ to slightly less than one third $\left(N_{A} / N_{T}=0.30 \pm 0.01\right)$ for $\mathrm{R} \sim 25^{\prime}$. This radial distance appears to be a preliminary plausible lower limit for the truncation radius, and indeed the same ratio in the two outermost radial bins attains smaller constant values. Note that the main vertical sequence partially overlapping with the acceptance region is almost entirely made up of field stars.

To further constrain the plausibility of the above working hypothesis concerning the radial extent of M92, we decided to investigate the radial distribution of extragalactic sources. We adopted the entire set of $r$-band images collected with MegaCam at CFHT and performed a new independent photometry by using Sextractor (Bertin \& Arnouts 1996). The non-point like sources were selected -following Evans et al. (2010)- as the objects with a local point spread function $90 \%$ enclosed counts fraction larger than $1.4^{\prime \prime}$. The adopted value was fixed by eye inspection of the mean $r$-band image. The Fig. 5] shows the radial distribution of the non-point like sources (blue dots) over the mean $r$-band image with apparent magnitudes between $r \sim 19$ and $r \sim 22$ mag. To help the eye to identify the sky area covered by M92, the red and the purple circles display the Wilson truncation radius based on both the number density and the surface brightness profile (see $\S 4$ ). The smaller orange circles show the candidate galaxy clusters identified from the SDSS DR6 (Wen et al. 2009). A glance at data plotted in this figure shows that the candidate galaxy clusters are located either close to the truncation radius or beyond it. The innermost candidate galaxy cluster appears a bit suspicious, since it is located in a cluster region with a high stellar density.

We selected the objects located inside the sky region covered by the five candidate galaxy clusters beyond the truncation radius and plotted them in the CMD and we found that a significant fraction $(\sim 65 \%)$ of them are located outside the acceptance region (blue triangles in panel c) of Fig. 4). This fraction agrees, within the errors, quite well with the ratio between accepted and total number of stars (see the discussion above in this section).

To remove spurious stars that were erroneously accepted, we used the method described by Walker et al. (2011) for the GC IC 4499. This method works particularly well for our datasets, due to the large sky area they cover. To estimate the density of the rejected stars, the sky area covered by CFHT $\left(R \lesssim 30^{\prime}\right)$ and by SDSS $\left(0.5^{\circ} \lesssim R \lesssim 2^{\circ}\right)$ data was divided into concentric annuli. The star counts based on SDSS data were corrected to account for the non homogeneous coverage of this photometric catalog. Panel a) of Fig. 6] shows the logarithmic surface density of these objects (number of rejected stars, $N_{R}$, per $\operatorname{arcmin}^{2}$ ) as a function of the inverse of the radial distance. We performed a linear fit to the individual points and by extrapolating to infinite radial distance we found that the asymptotic value is $\mu_{1}=0.42 \pm 0.10$ [logarithmic number of stars per $\left.\operatorname{arcmin}^{2}\right]$. We also estimated the asymptotic value as the mean of the five outermost values, finding $\mu_{2}=0.36 \pm 0.10$. We adopted the mean of the above estimates: $\mu=0.39 \pm 0.14$.

In panel b) of Fig. 6 we plotted the logarithm of the ratio between the number of accepted stars and the number of rejected stars $\left(N_{A} / N_{R}\right)$. This ratio was estimated using the entire dataset. To estimate the mean asymptotic value, we adopted the average of the outermost three radial bins, obtaining $\chi=-0.41 \pm 0.03$. Eventually, by multiplying the number of rejected stars per arcmin ${ }^{2}$ by $N_{A} / N_{R}$, we found the number of candidate field stars that were erroneously classified as candidate M92 stars. Notably, we found that the asymptotic number of spuriously accepted stars is $10^{\mu} \times 10^{\chi} \sim 0.95 \mathrm{star} / \mathrm{arcmin}^{2}$. By subtracting this value from the number of the $a c$ cepted stars per unit area, we obtained the final Count Catalog of the candidate M92 stars. This catalog was used to compute the Number Density (ND) radial profile. We divided the cluster into concentric annuli and we counted the number of stars per $\operatorname{arcmin}^{2}$ that fall inside each region, obtaining a radial profile ranging from $R \sim 1.5^{\prime \prime}$ out to $R \sim 2^{\circ}$. The error on each of the points is calculated as the square root of the number of stars, divided by the area of the annulus.

Following Walker et al. (2011), we measured the spurious stellar flux which affects the stellar luminosity of accepted stars. The logarithmic flux density of the rejected stars per $\operatorname{arcmin}^{2}\left(F l u x_{R}\right)$ and the ratio between 
the flux of accepted and rejected stars $\left(F l u x_{A} / F l u x_{R}\right)$ are plotted in panels c) and d) of Fig. 6. The logarithm of the surface flux density of the rejected stars approaches $\epsilon_{1}=-5.72 \pm 0.03$ [logarithmic star flux per arcmin ${ }^{2}$ ] when extrapolated to infinite radial distance, whereas it is $\epsilon_{2}=-5.76 \pm 0.03$ when we use the mean value of the last three points. The mean of these values is $\epsilon=-5.74 \pm 0.04$, whereas the logarithm of the ratio between accepted and rejected stars is $\xi=-0.82 \pm 0.07$. In this case, the flux of spurious accepted stars is $10^{\epsilon} \times 10^{\xi} \sim 0.310^{-6}$ Flux/arcmin ${ }^{2}$. By subtracting this last value from the accepted stellar flux density, we obtained an independent final Flux Catalog for the candidate M92 stars. This catalog was used to calculate the SB radial profile. As in the previous case we divided the cluster into annular regions, and the SB for each annulus was computed by adding the flux contribution of the stars located inside the annulus and by dividing for its area. Data plotted in panels b) and d) of Fig. 6 show that the ratio between the number of accepted and rejected stars is more robust than the ratio between the flux of accepted and rejected stars, since the intrinsic dispersion of the former one is at least a factor of two smaller than the latter one. The difference is caused by the fact that the number ratio is rooted in the radial distribution of MS stars, while the flux ratio traces the radial distribution of bright evolved stars. To further constrain the role of bright stars in determining the radial slope of the SB, we calculated two more SB profiles by considering only stars fainter than a limiting magnitude of $i=15$ (SB-15) and $i=17$ (SB-17) mag (see Sect. 4).

The error on the surface brightness profile was estimated by propagating the error on individual measurements of star magnitudes. The intrinsic photometric error, in the magnitude range adopted to estimate the surface brightness profile, is typically of the order of a few hundredths of a magnitude (see $\S 2$ ), because groundbased images were collected in good seeing conditions (DiC10) and we typically have more than ten images per band. The same applies for ACS images adopted in the central regions. We also calculated the error of the absolute photometric zero-points. Following DiC10, we calibrated the CFHT photometric catalog by using the local standards by Clem et al. (2007). The ACS and the SDSS photometric catalogs were also transformed into the same photometric systems by using the new local standards. We ended up with a mean calibration error in the $i$-band of $0.02 \pm 0.04 \mathrm{mag}$ (Di Cecco 2009). This error was eventually summed in quadrature with the intrinsic photometric error.

We evaluated the symmetry of the ND as a function of the radial distance by using the Count Catalog. To avoid possible systematic uncertainties in the radial distribution, the symmetry of the ND was estimated on the basis of ACS and CFHT data. The SDSS data were neglected since they do not uniformly cover the area of the sky around the cluster center. The conclusions concerning the departure from circular symmetry of the radial distribution for distances between $6^{\prime}$ and $30^{\prime}$ are not affected by the inclusion of the SDSS dataset. We computed the contour levels of the candidate M92 and field stars (black and red lines in panel a) of Fig. 7). The contour levels become less circular symmetric when moving toward the outermost regions, and for radial distances between $6^{\prime}$ and $10^{\prime}$ the stellar density decreases by almost one order of magnitude (see top panels of Fig. 7). The contour levels become asymmetric at a distance of $\sim 13^{\prime}$ (see panel a) of Fig. (7), which is almost equivalent to the truncation radius of M92 (see column 5 in Table 1) available in the literature. Outside this region the distribution of candidate cluster stars becomes clumpy. Panels b) and c) of Fig. 7] show the projected linear density (marginal) along the axes of the candidate M92 and field stars (black and red lines). By inspecting these panels, we notice that the detected sharp decrease in density is associated with the radial distance at which the surface density of the candidate M92 stars becomes smaller than that of the candidate field stars.

To trace in detail the departure of the contour levels from circular symmetry, we performed a fit of each contour with a circle. The center of the circles is identical to the center of the contours -i.e. the cluster center- and the radius of the circles is the fitting parameter. Then, we computed the residuals in arcsec between individual contours and best fitting circles. The residuals were estimated from the very center of the cluster out to a radial distance of $R \sim 800^{\prime \prime}$.

The residuals plotted in panel d) of Fig. 7] show that the innermost contour levels display symmetric radial distributions, and indeed the residuals attain vanishing values out to $R \sim 3^{\prime}$. At larger radial distances, the asymmetry increases out to $R \sim 9^{\prime}-10^{\prime}\left(R \sim 500-600^{\prime \prime}\right)$, where the residuals show a shoulder clearly connected with the density drop detected in the contour plot. At even larger distances the contours become more asymmetric (residuals $\sim 26^{\prime \prime}$ for distances of $R \sim 13^{\prime}$ ); the fit in the outermost regions fails to converge because of the large asymmetries. The increase in asymmetry that we found in the outer regions could be the consequence of the fluctuations associated with the decrease in density. To validate this working hypothesis we performed a series of simulations by using the observed density profile to compute synthetic GCs; the radial distribution of the synthetic GCs was required to be symmetric. We applied to these GCs the same procedure to evaluate the contour levels and the same fit with circles of variable radius. The vertical hatched area plotted in panel d) of Fig. 7 marks the residuals calculated for the synthetic GCs. The comparison between this area and the plotted points indicates that the asymmetry in the real cluster is at least $3 \sigma$ larger than in the synthetic clusters. To characterize further the nature of the asymmetries in the contour levels, we show two green arrows, in the top panel of Fig. 7] indicating the direction of the Galactic center (long arrow) and of the M92 proper motion (short arrow) according to Dinescu et al. (1999). We found no clear correlation between these directions and the clumpy distribution of candidate M92 stars at large radial distances.

Evidence of a clumpy stellar distribution in the outskirts of M92 was also present in the stellar density maps provided by Testa et al. (2000, see their Fig. 6), by Lee et al. (2003, see their Fig. 12) and by Jordi \& Grebel (2010, see their Fig. 17). The above results support the findings by Lee et al. (2003), concerning the marginal evidence of an elongation of outermost clumpy stars in the direction orthogonal to the direction of the Galactic center. 


\section{DYNAMICAL MODELS AND FITS}

We carried out fits of dynamical models to the observed radial profiles. We considered the King (1966) and the Wilson (1975) spherical and isotropic dynamical models, defined by the following distribution functions:

$$
\begin{gathered}
f_{\mathrm{K}}=A\left(e^{-a E}-e^{-a E_{0}}\right) \quad E \leq E_{0}, \\
f_{\mathrm{W}}=A\left\{e^{-a E}-e^{-a E_{0}}\left[1-a\left(E-E_{0}\right)\right]\right\} \quad E \leq E_{0} .
\end{gathered}
$$

The quantity $E$ is the specific star energy $E=v^{2} / 2+$ $\Phi(r)$, where $\Phi(r)$ is the mean-field gravitational potential, to be determined from the Poisson equation. Both distribution functions vanish for energies larger than the threshold energy $E_{0}$, corresponding to stars to be considered as unbound. The energy truncation can be translated into a truncation radius, $r_{t}$, which indicates the boundary of the system. For each family of models, the constants $A, E_{0}$, and $a$ in Eqs. (11) and (2) define two dimensional scales (a typical radius and a typical mass or velocity) and one dimensionless parameter, the central depth of the potential well (related to the concentration parameter). We recall that fitting by a one-component dynamical model assumes that the underline stellar populations are distributed homogeneously. To identify the best-fit model we adopted the procedure described by Zocchi et al. (2012). The results are shown in Figs. 8 and 9 the values of the relevant parameters of the bestfit models are listed in the upper part of Table 1.

The fit to the ND profile is shown in the top panel of Fig. 8, Both King and Wilson models underestimate the central ND profile, failing with respect to the four innermost points. A quantitative interpretation of this discrepancy remains unavailable, but the problem is likely to be related to the failure of the assumptions at the basis of a one-component dynamical model in the central regions. Concerning the King models, the good agreement with observations that can be found in the middle part of the profile breaks down around $700^{\prime \prime}$, where the profile approaches the background level $\left(0.95 \mathrm{star} / \operatorname{arcsec}^{2}\right)$. In the case of the Wilson models, instead, only the two outermost points are discrepant, and the model fits the data out to a distance greater than $1000^{\prime \prime}$. Note that the two outermost points are likely to be affected by errors in the subtraction of background stars. The bottom panel of Fig. 8 shows the fits to the SB profile. When compared to the King best-fit model, the Wilson bestfit model provides a more adequate overall description, not only in relation to the outermost points, as in the ND profile, but also in the central part of the profile. The satisfactory performance of the Wilson models indicates that the observations can be explained by means of a less abrupt truncation radius, with no need to introduce extra-tidal halos. In this case, the two outermost points were not taken into account to calculate the bestfit parameters, since they are expected to be even more affected by errors in the subtraction of background stars, compared to the corresponding points in the ND profile. In the figure, the background level (25.30 mag/arcsec $\left.{ }^{2}\right)$ is indicated as a horizontal dashed line.

Surprisingly, even if the ND and the SB profiles come from the same set of observations, the best-fit parameters determined by the fits are significantly different. We argue that this behavior is due to the fact that each profile represents a different aspect of the density distribution of the cluster. On the one hand, the ND profile, derived by considering the radial distribution of both luminous and faint stars, is dominated by the MS stars, which greatly outnumber evolved (RGB, HB) stars (Castellani et al. 2007). On the other hand, the SB profile is heavily affected by the presence of the brighter RGB stars. The difference in the best-fit models reflects the intrinsic difference in the radial distribution of the stellar tracer that determines each profile. This behavior should be interpreted as a signature of mass segregation 17 . Indeed, the evolved slightly more massive star 18 appear to be more centrally concentrated compared to stars with lower masses (see the values of the concentration parameter $c$ in Table 1).

This interpretation is confirmed by an additional test. We carried out the same fitting procedure on the SB-15 and the SB-17 profiles (as defined in Sect. 3). The resulting parameters are listed in the third and fourth rows of Table 1. The fits are shown in the top and in the bottom panel of Fig. 9] respectively. Inspecting the values of the best-fit parameters, it appears that by eliminating the brightest stars, the profiles tend to approach the ND profile. Indeed, the values of the concentration parameter and of the radial scale $-r_{s}$ - follow a monotonic trend from the SB profile to the SB-15, to the SB-17 and finally to the ND profile. Interestingly enough, for the SB-15 and SB-17 profiles, the King models reproduce the data better than the Wilson models, in contrast to what we found for the previously described ND and SB profiles. A reason for this can be found by comparing the radial extent of the different profiles. The three surface brightness profiles are shaped by the fact that the cut of candidate cluster stars brighter than a limiting magnitude causes a decrease in the radial extent and a more abrupt truncation of the profile. Indeed, the outermost radial point for the SB-15 profile is located at $R \sim 700^{\prime \prime}$, and for the SB-17 profile is located at $R \sim 500^{\prime \prime}$. The larger radial extent of the SB profile is the consequence of a few bright giants that keep the SB profile well above the background level.

For comparison, the middle part of Table 1 lists the most recent best-fit parameters published for M92. Note that in only a few cases the Wilson parameters are available. The acronyms in the first column identify the papers in which the results have been published.

The lower part of Table 1 lists the results of the fits to the other two 19 available surface brightness profiles in the literature, the one by T95, and the one by L03. We decided to add to both datasets the surface brightness data by Noyola et al. 2006 (N06), which are relative to the innermost region of the cluster. The results of these fits are shown in Fig. 10. For the T95+N06 profile, the models provide an equally good fit to the data; we note that in this case the outermost point in the profile is located at a radial distance $\mathrm{R}<700^{\prime \prime}$. For the L03+N06 profile, the good agreement with observations that is seen in the inner and middle parts of the profile breaks down

\footnotetext{
17 The occurrence of mass segregation in M92 was also suggested by Andreuzzi et al. (2000).

18 Note that hot HB stars are less massive than MSTO stars, but they are a minor fraction of evolved cluster stars.

19 Another surface brightness profile was presented by JG10, but the dataset is not publically available.
} 
around $600^{\prime \prime}$ for the King model, and at more than $1000^{\prime \prime}$ for the Wilson model. We recall that a similar behavior was also found for the fit to the ND profile previously described. In the last profile the outermost points are not reproduced by any of the considered models.

The values of the parameters listed in Table 1 are not consistent with each other, within the errors. In conclusion, we believe that a proper comparison of the values of the best-fit parameters found by fitting models to different (ND, SB) profiles requires that we take into account the role of the different stellar tracers in determining their shape, which makes standard one-component dynamical models questionable.

\section{DISCUSSION AND FINAL REMARKS}

We studied the radial distribution of stars of the globular cluster M92 by using ground-based (MegaCam at CFHT, SDSS) and space (ACS on HST) data.

The contour levels, based on star count data, are symmetric in the innermost regions, and exhibit an increasing asymmetry for radial distances between $3^{\prime}$ and $9^{\prime}$ $10^{\prime}$. For distances larger than $\sim 13^{\prime}$ the stellar distribution becomes clumpy. The contour levels do not exhibit a preferred orientation in space. We calculated two independent radial profiles, to describe the distribution of stars in the cluster, the number density (ND) and the surface brightness (SB) profile. To calculate these profiles, we subtracted the background contamination with two independent methods. We performed fits of spherical King and Wilson models to the above profiles. Wilson models appear to reproduce, better than King models, the behavior of the outermost regions of the cluster with no need of extra-tidal halos. Interestingly, for the ND profile, both models significantly underestimate the observations in the innermost regions.

We also found that the best fit to the ND and to the SB profile are provided by two different models for the two families, even though the profiles are derived from the same datasets. We argue that this difference is caused by a difference in the radial distribution of the stellar tracers that characterize the two observed profiles. The ND profile traces the radial distribution of MS stars, whereas the SB profile that of bright evolved (RGB, HB) stars. This conclusion is supported also by the results of a test that has been carried out on two additional profiles, calculated by considering only stars fainter than a given magnitude.

Hopefully, a thorough discussion of the behavior in this and other clusters should determine which of the various profiles considered is best suited for a study in terms of one-component models. To our knowledge, this is the first investigation in which independent estimates of both ND and SB profiles are provided, starting from the same set of data, and compared.

In this context a key role can be played by the new generation of wide field imagers that are available at the 4-8m class telescopes (Dark Energy Camera and Survey at the CTIO 4m Blanco telescope, Mohr et al. 2012; Hyper SuprimeCam at SUBARU20). In a single pointing they can cover the entire extent of a large number of GCs and with modest exposure times will allow us to perform homogeneous and accurate photometry several magnitudes fainter than the MSTO.

It is a real pleasure to thank an anonymous referee for his/her positive opinion concerning the content and the cut of our paper and his pertinent suggestions that helped us to improve its readibility. This work was partially supported by PRIN-INAF 2011, 'Tracing the formation and evolution of the Galactic halo with VST' (P.I. M. Marconi), by PRIN-MIUR (2010LY5N2T) 'Chemical and dynamical evolution of the Milky Way and Local Group galaxies' (P.I.: F. Matteucci), by the IAC (grants, 310394) and by the Education and Science Ministry of Spain (grants AYA2010-16717). One of us, A. Di Cecco, thanks the warm hospitality of the Dominion Astrophysical Observatory for the stay during which part of this work has been done; G. Bono thanks ESO for support as a science visitor. This publication makes use of data products from VizieR (Ochsenbein et al. 2000) and from the Two Micron All Sky Survey, which is a joint project of the University of Massachusetts and the Infrared Processing and Analysis Center/California Institute of Technology, funded by the National Aeronautics and Space Administration and the National Science Foundation. We also used the NASA/IPAC Extragalactic Database (NED) which is operated by the Jet Propulsion Laboratory, California Institute of Technology, under contract with the National Aeronautics and Space Administration.

\section{REFERENCES}

Aguilar, L., Hut, P., \& Ostriker, J. 1988, ApJ, 335, 720

Aihara, H., Allende Prieto, C., An, D., et al. 2011, ApJS, 193, 29

Andreuzzi, G., Buonanno, R., Fusi Pecci, F., Iannicola, G., \& Marconi, G. 2000, A\&A, 353, 944

Bertin, E,\& Arnouts, S. 1996, A\&AS, 117, 393

Castellani, V., Calamida, A., Bono, G., et al. 2007, ApJ, 663, 1021

Chun, S.-H., Kim, J.-W., Sohn, S. T. et al. 2010, AJ, 139, 606

Clem, J. L., Vanden Berg, D. A., \& Stetson, P. B. 2007, AJ, 134, 1890

Correnti, M., Bellazzini, M., Dalessandro, E. et al. 2011, MNRAS, 417,2411

Di Cecco, A. 2009, Deep wide-field photometry of the Galactic globular cluster M92, Ph.D. Thesis, Università di Roma Tor Vergata

Di Cecco, A., Becucci, R., Bono, G. et al. 2010, PASP 122, 991 [DiC10]

Dinescu, D. I., Girard, T. M., \& van Altena W. F. 1999, AJ, 117, 1792

${ }^{20}$ http://www.naoj.org/Projects/HSC/index.html
Evans, I. N., Primini, F. A., Glotfelty, K. J., et al. 2010, ApJS, 189, 37

Gnedin, O. Y.,\& Ostriker, J. P. 1997, ApJ, 474, 223

Grillmair, C. J., Freeman, K. C., Irwin, M., \& Quinn, P. J. 1995, AJ, 109,2553

Grillmair, C. J., \& Johnson, R. 2006, ApJ, L639, 17

King, I. R., 1962, AJ, 67, 471

King, I. R., 1966, AJ, 71, 64

Jordi, K., \& Grebel, E. K. 2010, A\&A, 522, 71 [JG10]

Lee, K. H., Lee, H. M., Fahlman, G. G. et al. 2003, AJ, 126, 815 [L03]

Leon, S., Meylan, G.. \& Combes, F. 2000, A\&A, 359, 907

Lehmann, I., \& Scholz, R. D. 1997, A\&A, 320, 776

McLaughlin, D. E., \& van der Marel, R. P. 2005, ApJS, 161, 304 [MLvdM05]

Meylan, G..\& Heggie, D. C. 1997, A\&ARv, 8, 1

Mohr, J. J. et al. 2012, SPIE, submitted, arXiv1207.3189

Noyola, E., \& Gebhardt, K. 2006, AJ, 132, 447 [N06]

Ochsenbein, F., Bauer, P., \& Marcout, J. 2000, A\&AS, 143, 221 
Odenkirchen, M., Grebel, E. K., Rockosi, C. M. et al. 2001, ApJ, L548, 165

Odenkirchen, M., Grebel, E. K., Dehnen, W. et al. 2003, AJ, 126 2385

Olszewski, E. W., Saha, A., Knezek, P. et al. 2009, AJ, 138, 1570

Spitzer, L. Jr. 1958, ApJ, 127, 544

Spitzer, L. Jr., \& Chevalier, R. A. 1973, ApJ, 183, 565

Spitzer, L. Jr., \& Harm, R. 1958, ApJ, 127, 544

Stetson, P. B. 1987, PASP, 99, 191

Stetson, P. B. 1994, PASP, 106, 250
Stetson, P. B., Bruntt, H., \& Grundahl, F. 2003, PASP, 115, 413

Testa, V., Zaggia, S. R., Andreon, S. et al. 2000, A\&A, 356, 127 [T00]

Trager, S. C., King, I. R., \& Djorgovski, S., 1995, AJ, 109, 218 [T95]

Walker, A. R., Kunder, A. M., Andreuzzi, G. et al. 2011, MNRAS, 415,643

Wen, Z. L., Han, J. L., \& Liu, F. S., 2009, ApJS, 183, 197

Wilson, C. P., 1975, AJ, 80, 175

Zocchi, A., Bertin, G., \& Varri, A. L. 2012, A\&A, 539, 65 
TABLE 1

Best-Fit PARAmeters.

\begin{tabular}{|c|c|c|c|c|c|c|c|c|}
\hline \multirow[t]{2}{*}{ Profile } & \multicolumn{4}{|c|}{ King models } & \multicolumn{4}{|c|}{ Spherical Wilson models } \\
\hline & $\Psi$ & $\mathrm{c}$ & $r_{\mathrm{s}}$ & $r_{t}$ & $\Psi$ & $\mathrm{c}$ & $r_{\mathrm{s}}$ & $r_{t}$ \\
\hline ND & $6.91 \pm 0.02$ & $1.50 \pm 0.01$ & $34.25 \pm 0.38$ & $18.11 \pm 0.44$ & $5.84 \pm 0.02$ & $1.73 \pm 0.01$ & $48.03 \pm 0.42$ & $42.63 \pm 1.19$ \\
\hline SB & $8.40 \pm 0.01$ & $1.95 \pm 0.00$ & $15.22 \pm 0.02$ & $22.80 \pm 0.18$ & $6.65 \pm 0.01$ & $2.14 \pm 0.00$ & $21.08 \pm 0.05$ & $48.80 \pm 0.85$ \\
\hline SB-15 & $7.20 \pm 0.01$ & $1.59 \pm 0.00$ & $19.43 \pm 0.05$ & $12.56 \pm 0.10$ & $6.52 \pm 0.01$ & $2.06 \pm 0.00$ & $24.33 \pm 0.06$ & $46.67 \pm 0.42$ \\
\hline SB-17 & $6.95 \pm 0.01$ & $1.51 \pm 0.00$ & $23.17 \pm 0.06$ & $12.63 \pm 0.12$ & $6.29 \pm 0.01$ & $1.94 \pm 0.00$ & $29.04 \pm 0.06$ & $41.97 \pm 0.53$ \\
\hline T95 & 7.92 & 1.81 & 23.67 & 15.20 & & & & \\
\hline T00 & & & & 12.33 & & & & \\
\hline L03 & 8 & 1.83 & 12.42 & 14.00 & & & & \\
\hline MLvdM05 & 7.5 & 1.68 & 16.15 & 12.88 & 5.9 & 1.75 & 26.51 & 24.85 \\
\hline JG10 & 6.93 & 1.51 & 23.37 & 12.55 & & & & \\
\hline T95+N06 & 7.54 & 1.69 & 14.72 & 12.07 & 6.34 & 1.96 & 19.33 & 29.58 \\
\hline $\mathrm{L} 03+\mathrm{N} 06$ & 7.84 & 1.78 & 13.46 & 13.67 & 6.61 & 2.12 & 18.94 & 41.72 \\
\hline
\end{tabular}

Note. - For each model, we list the dimensionless parameter $\Psi$, the concentration $c$, the scale radius $r_{s}$ (arcsec), and the truncation radius $r_{t}$ (arcmin). The different profiles are identified by the label in the first column. The cases indicated as T95+N06 and L03+N06 refer to the fits we performed on composite profiles, obtained by combining the profiles from T95 and L03 with the profile from Noyola et al. (2006, [N06]), which covers the innermost regions of the cluster. 

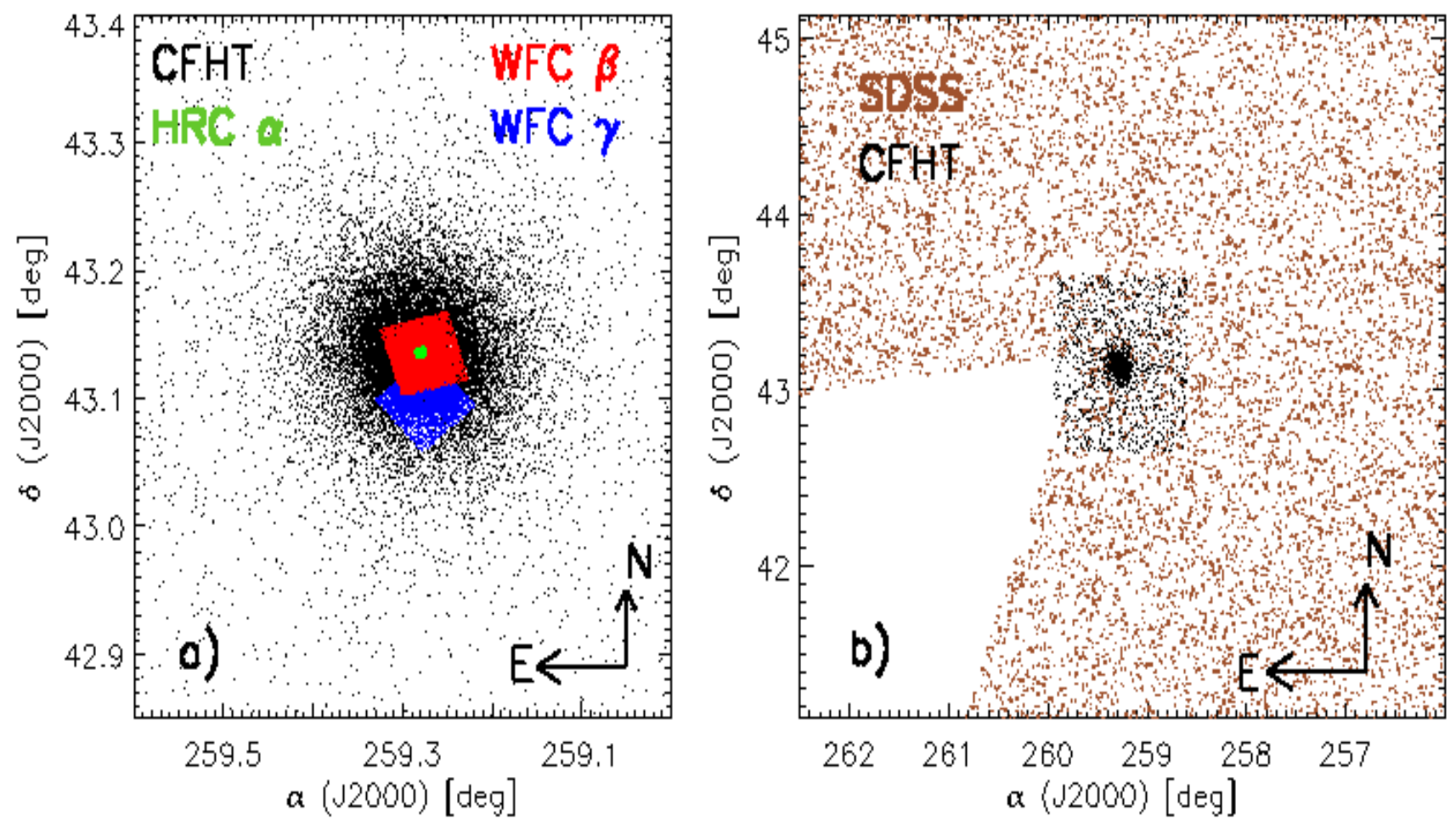

FIG. 1. - Panel a) - area of the sky across the globular cluster M92 covered by the different sets of space images collected with the Advanced Camera for Surveys (ACS) on board the HST. The field of view of the sky plot is $1^{\circ} \times 1^{\circ}$. The red and the blue squares show the images collected with the Wide Field Channel (WFC, pointings $\beta, \gamma$ ), while the green square those collected with the High Resolution Channel (HRC, $\alpha$ ). The black dots display the photometric catalog based on ground-based images collected with CFHT. The orientation is shown in the bottom right corner. Panel b) - same as Panel a), but for datasets collected with ground-based telescopes, namely CFHT (black dots) and SDSS (brown dots). Note that the latter dataset does not uniformly cover the area of the sky around M92. The field of view of the sky plot is $4^{\circ} \times 4^{\circ}$. See text for more details. 

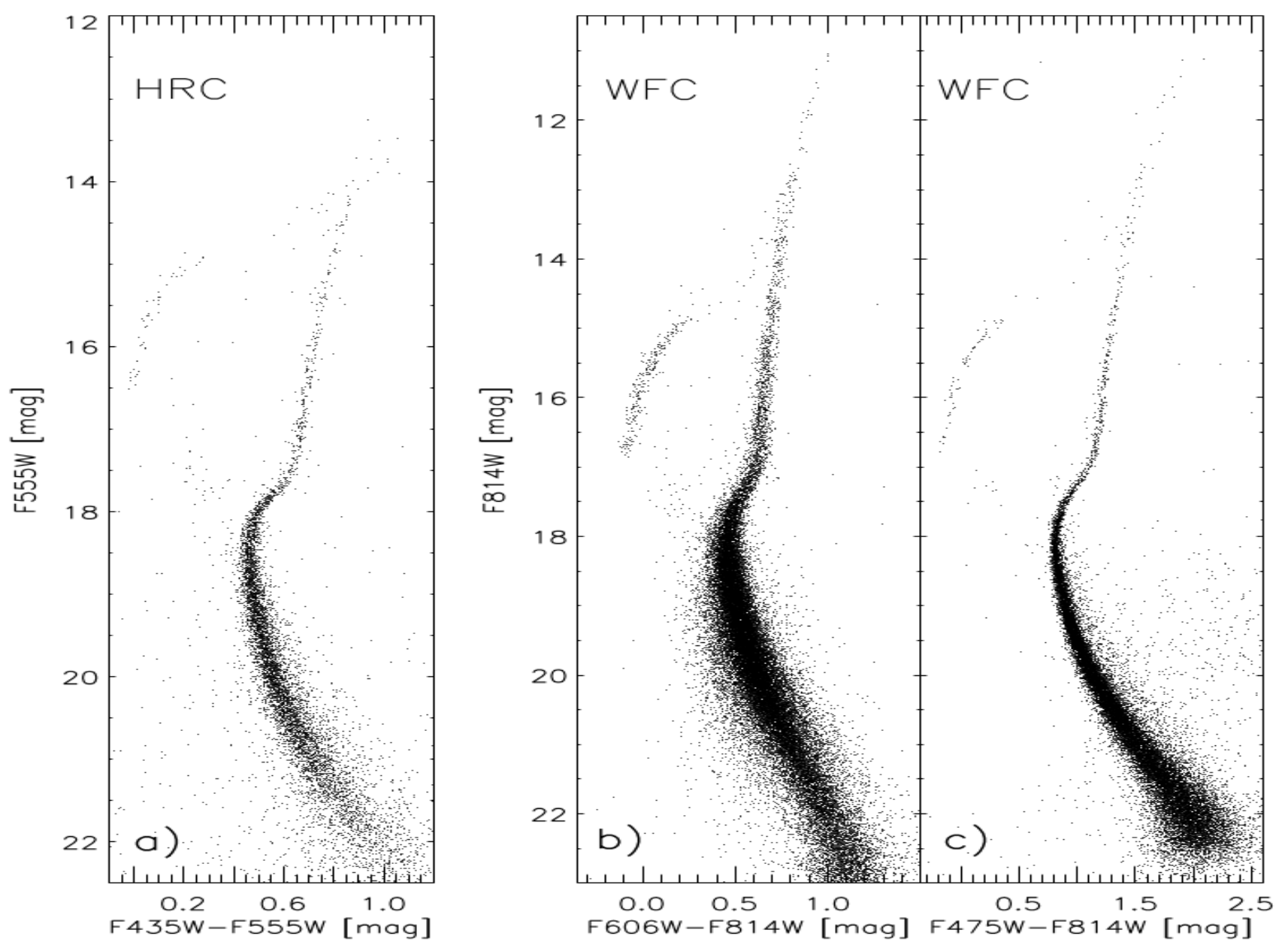

FIG. 2.- CMDs of M92 based on ACS at HST, pointings $\alpha, \beta$, and $\gamma$ in panels a), b), and c), respectively. Stars plotted in these CMDs were selected according to different selection criteria. Data of pointing $\gamma$ are the same as in DiC10. 


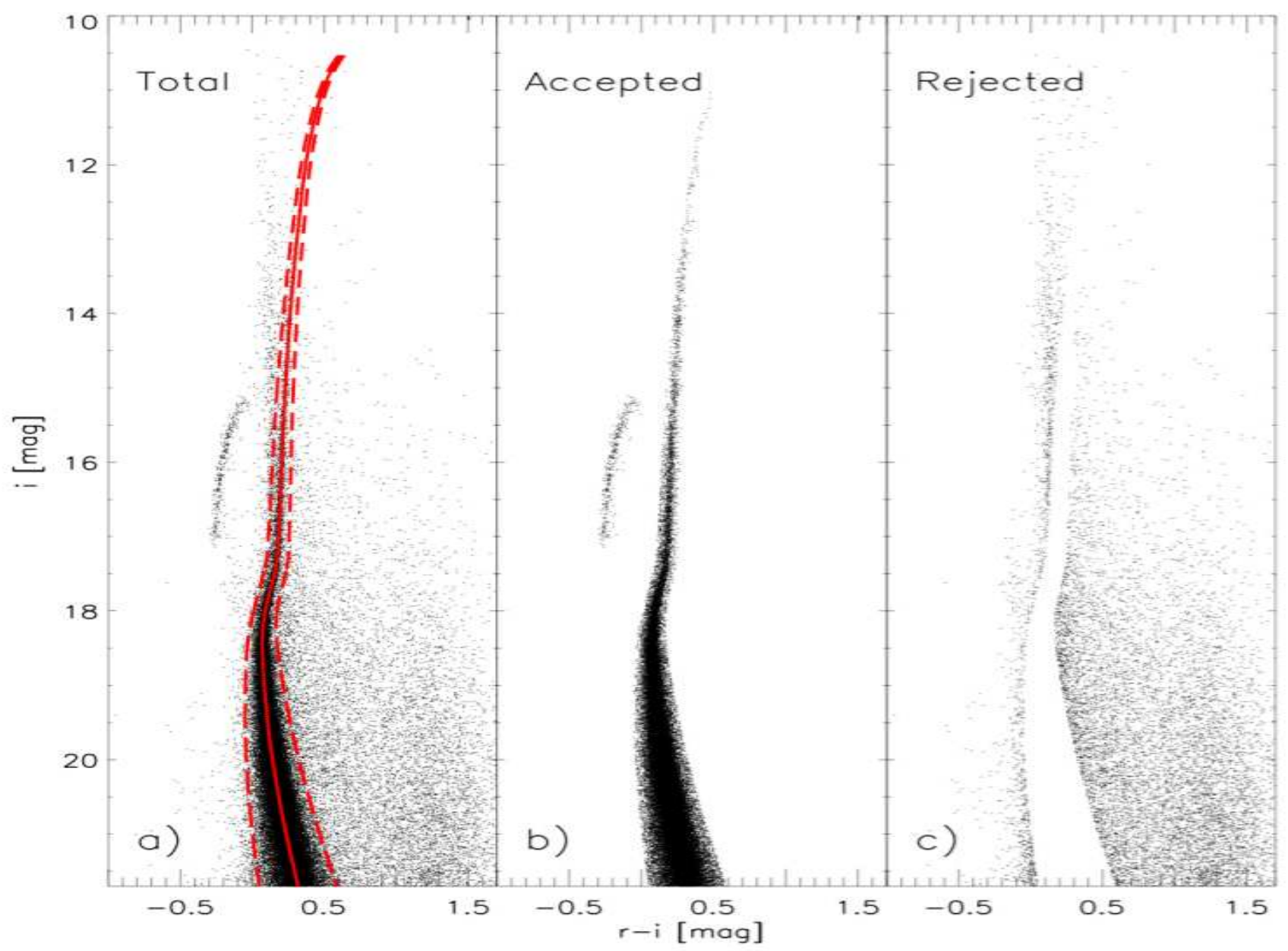

FIG. 3.- Panel a) $i, r-i$ CMD based on both ground-based (MegaCam at CFHT) and space (ACS-WFC at HST) data. Panels b) and c): CMDs for candidate M92 (accepted) and candidate field (rejected) stars, respectively. See text for more details. 


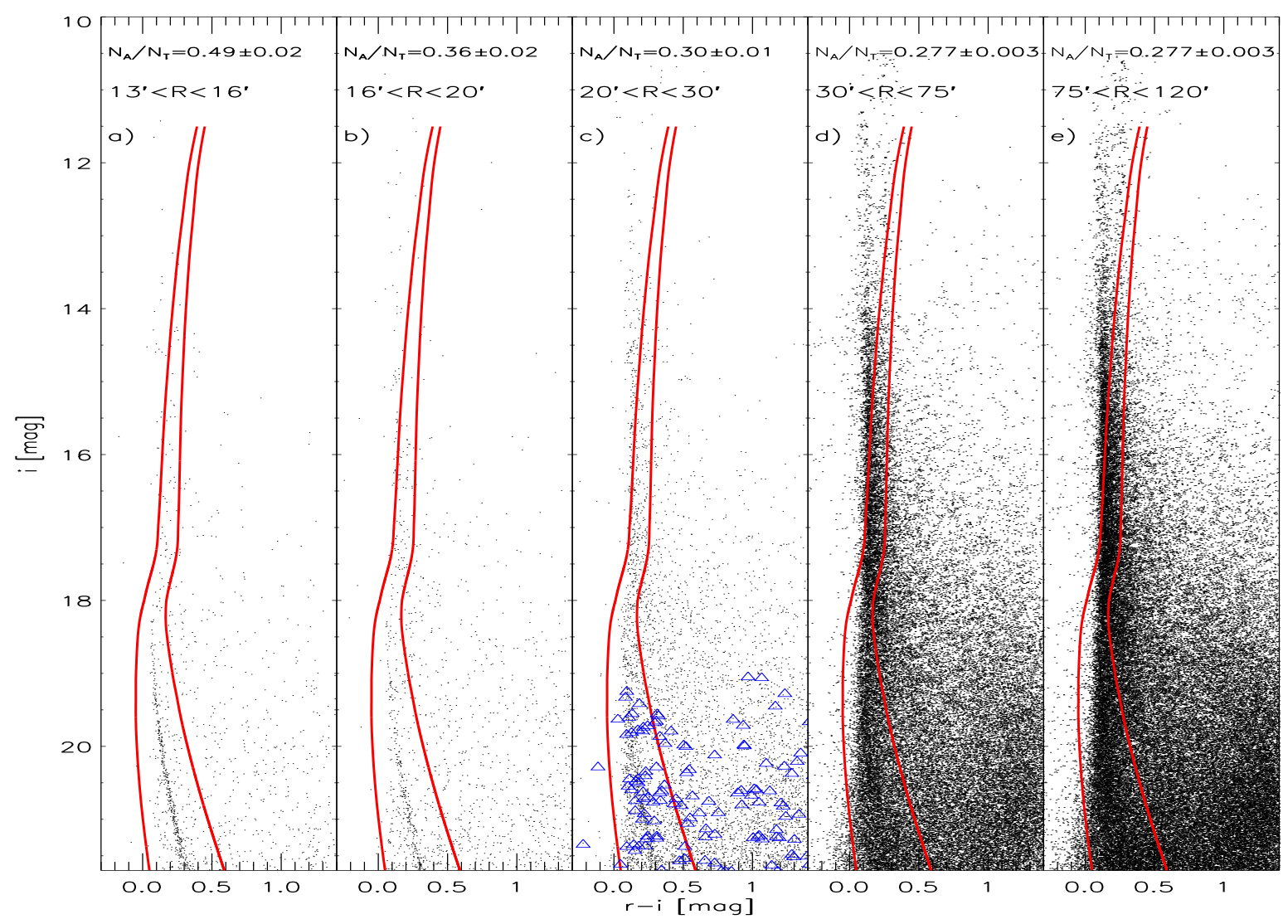

FIG. 4.- Panel a) - i,r-i CMD based on MegaCam/CFHT data. The red solid lines display the acceptance region defined in Fig. 3 The radial extent of the bin and the ratio between candidate cluster stars and total number of stars are also labeled. Panels b) and c) Same as for panel a), but for stars located at larger radial distances. The blue triangles plotted in panel c) are the objects located inside the candidate galaxy clusters (Wen et al. 2009). See text for more details. Panels d) and e) - same as for panel a), but for stars entirely located inside the SDSS dataset. 


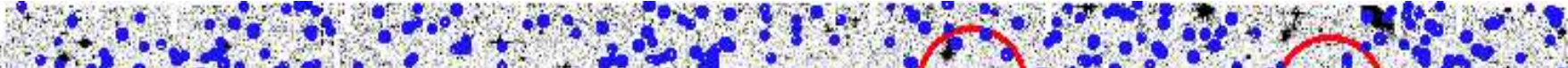

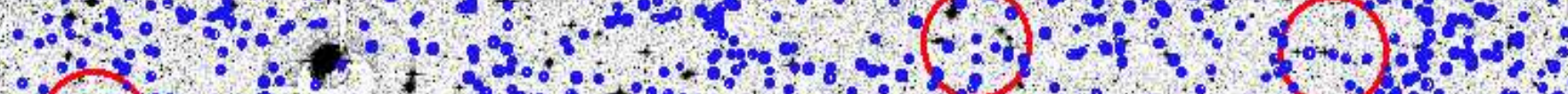

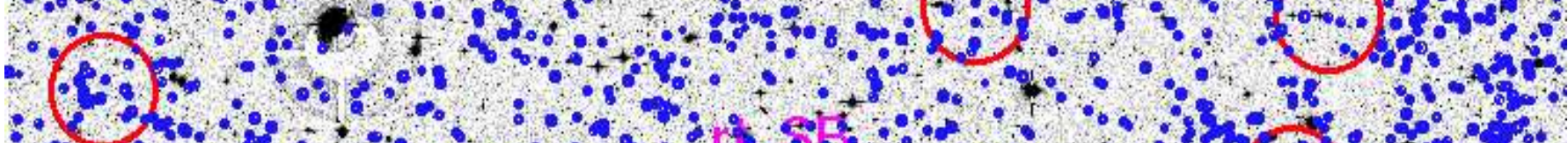

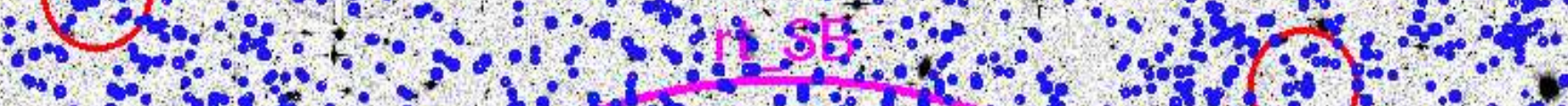

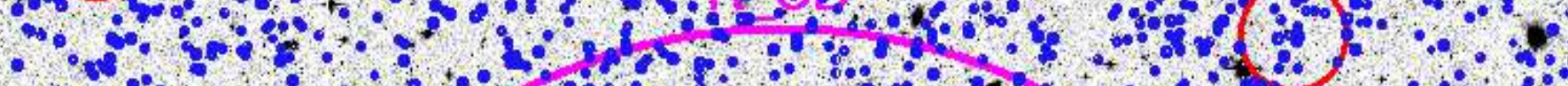

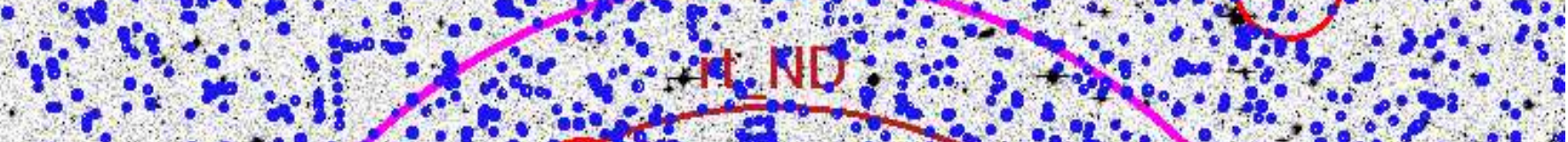

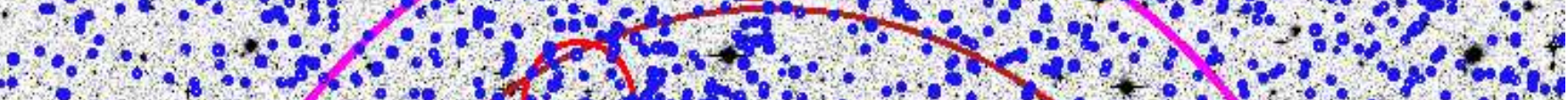

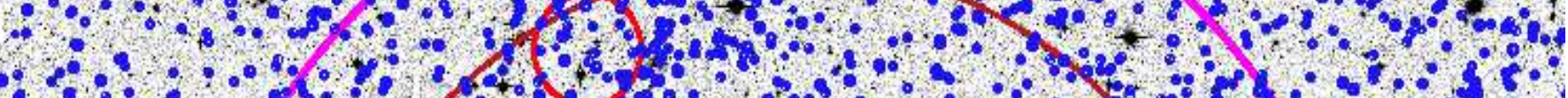

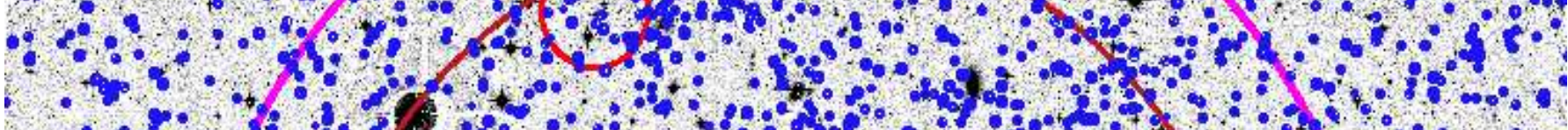

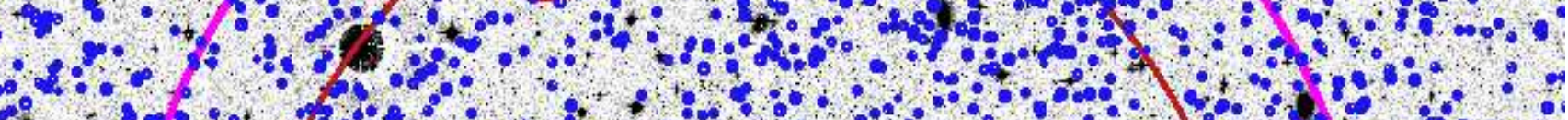

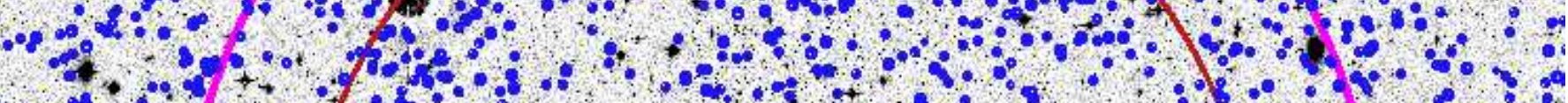

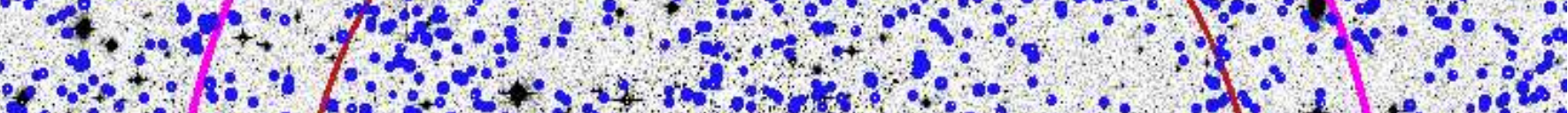

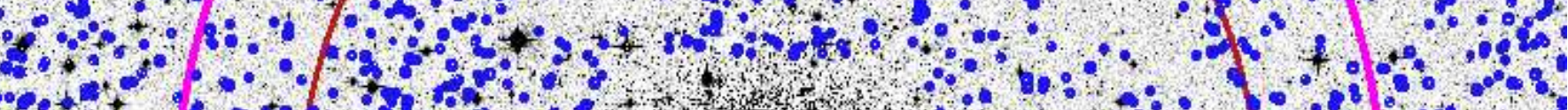

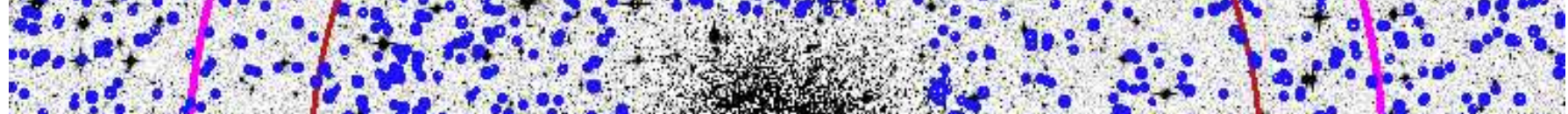

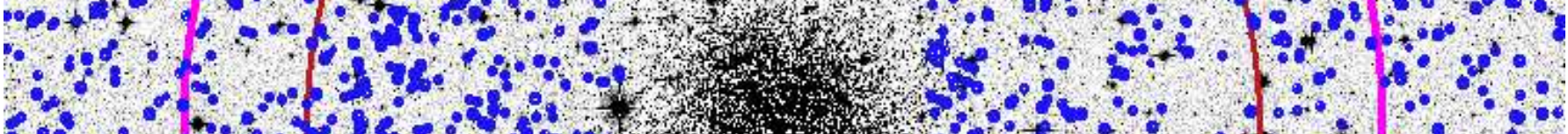

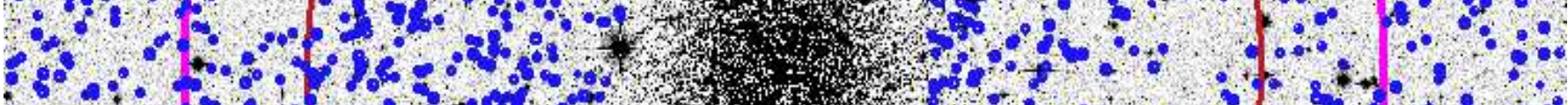

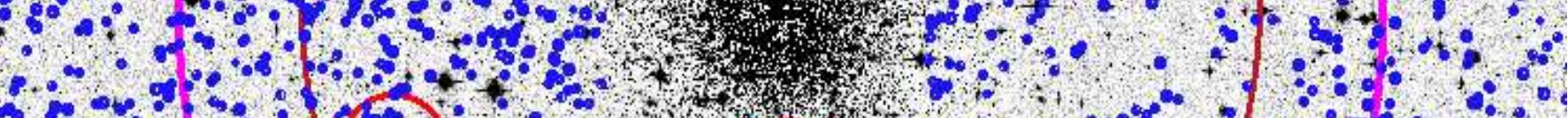

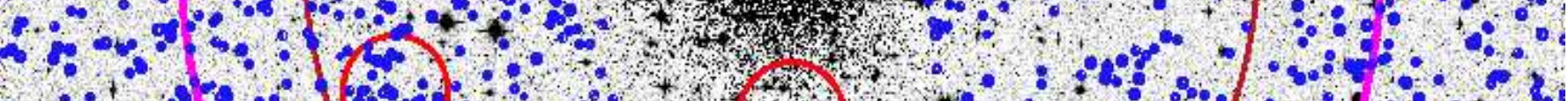

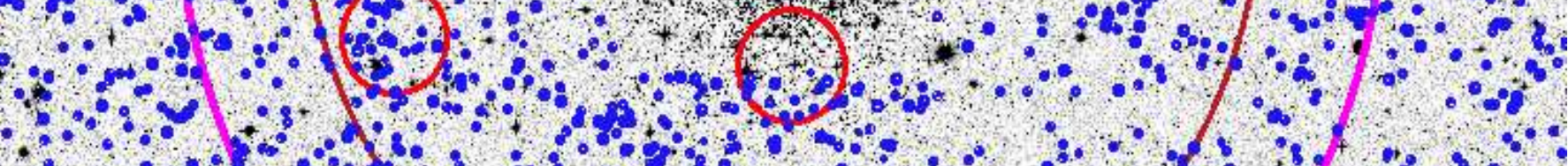

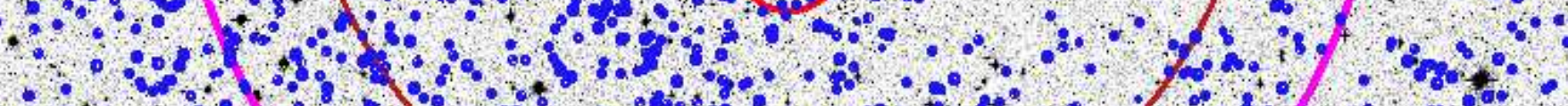

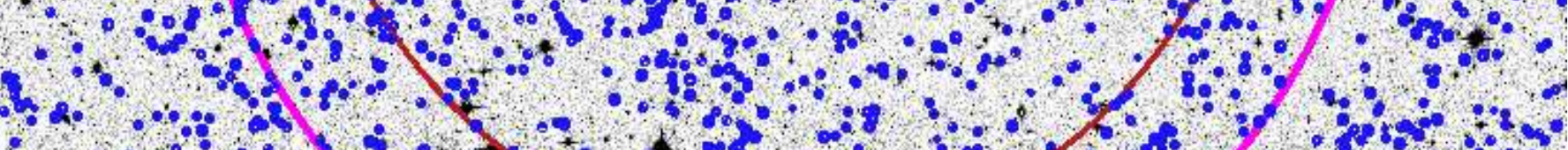

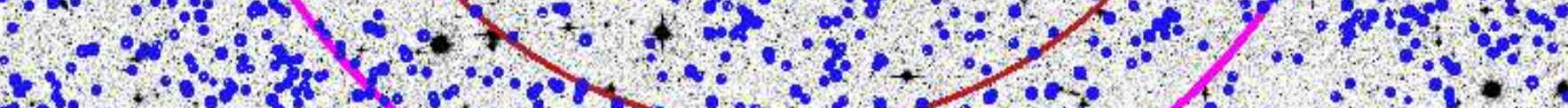

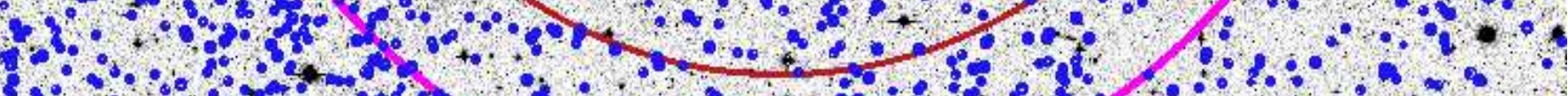

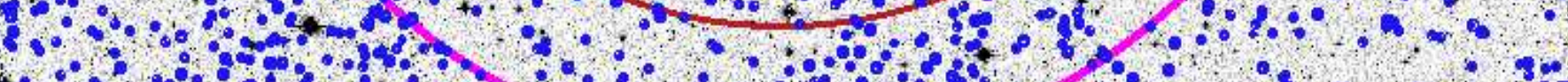

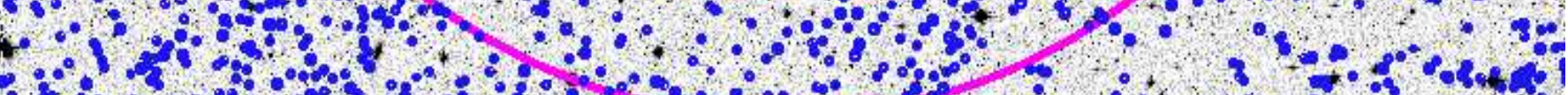

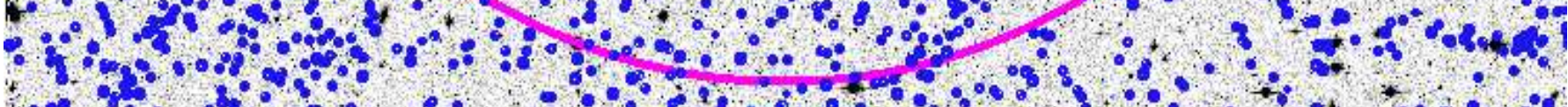

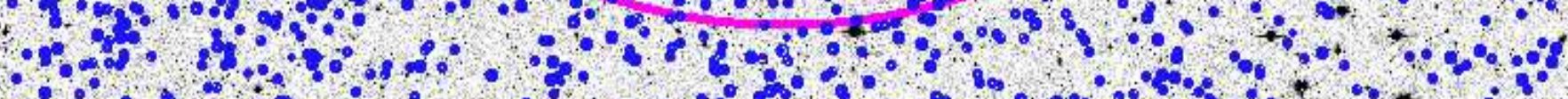

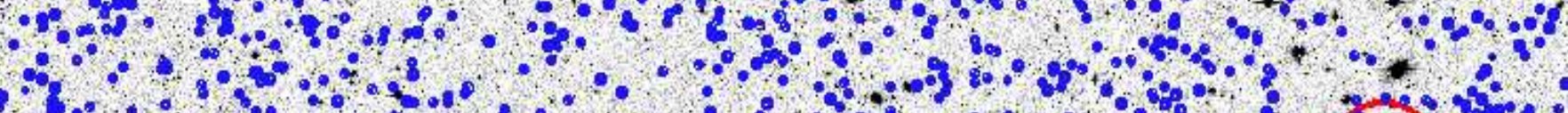

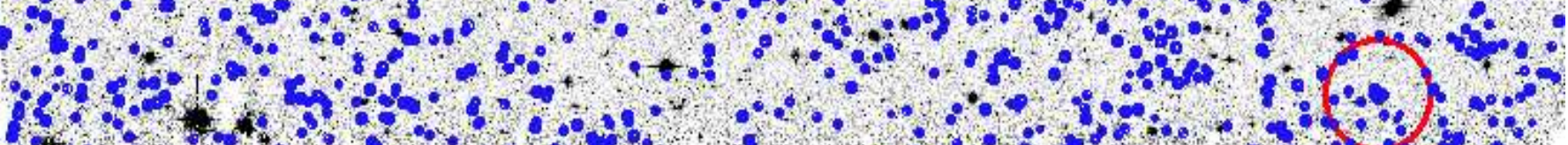

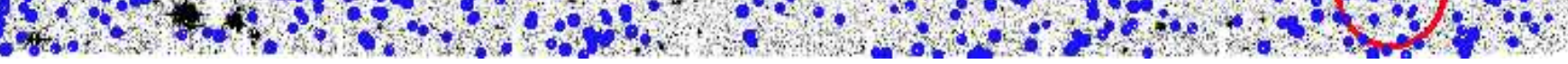

Fig. 5. - M92 $r$-band image of the CFHT dataset. The blue dots display the non-point like sources identified using Sextractor. The large purple and red circles display the Wilson truncation radius according to the SB and the ND profile. The small orange circles show the candidate galaxy clusters identified by Wen et al. (2009). The orientation is: North up and East left, the field of view is $1^{\circ} \times 1^{\circ}$. 

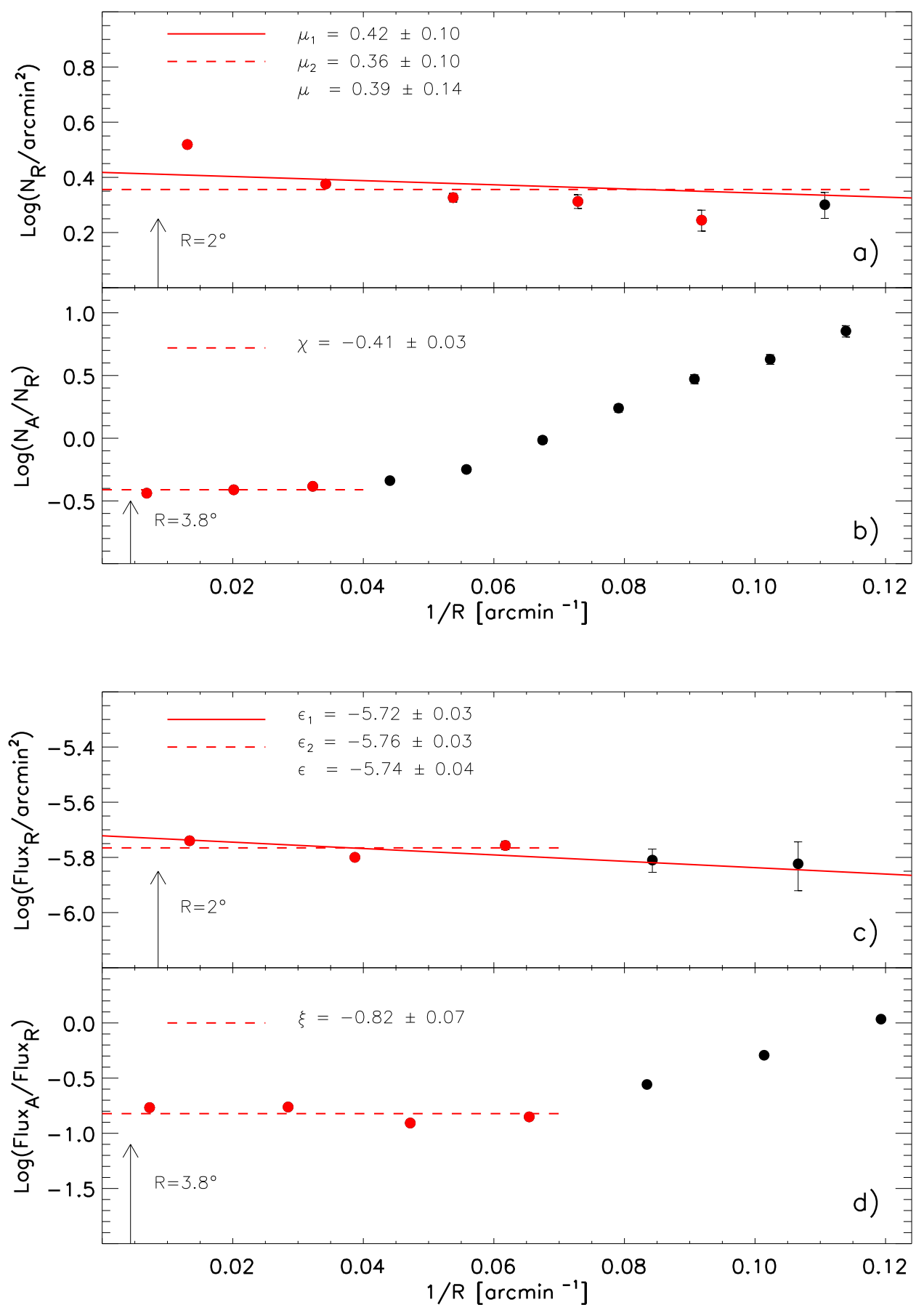

FIG. 6.- Panels a) and b): logarithm of the number density of the rejected stars and of the ratio between the number of accepted and rejected $-N_{A} / N_{R}-$ stars as a function of the inverse of the radial distance. Panels c) and d): logarithm of the flux density of the rejected stars, and of the ratio between the flux of accepted and rejected - Flux $_{A} / F_{l u x_{R}}$ - stars. The vertical arrows display the edge of outermost annulus, the solid lines show the linear fits over the entire samples, the dashed lines indicate the mean of the outermost annuli (red points). See text for more details. 

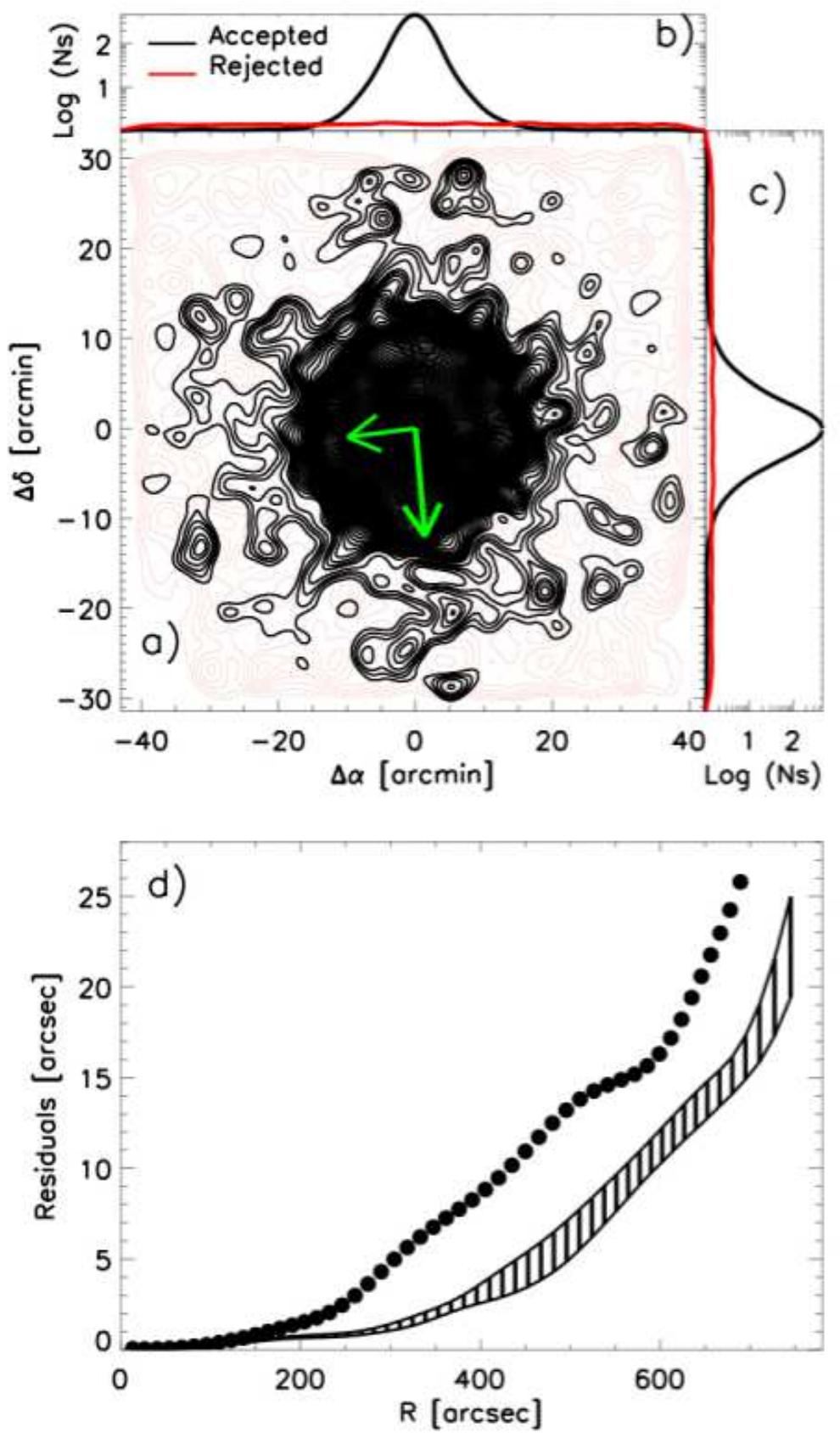

FIG. 7.- Panel a): contour levels for candidate M92 (black) and field (red) stars projected onto the sky. The long green arrow marks the direction of the Galactic Center, the short one the M92 proper motion. Panels b) and c): projected logarithmic distribution (marginal) along the horizontal and the vertical axis for candidate M92 (black) and field (red) stars. Panel d): the black filled circles show the residuals of the fits to the contour levels plotted in panel a) with circles of variable radius as a function of the radial distance. The vertically hatched area shows the results of simulations (see text for more details). 


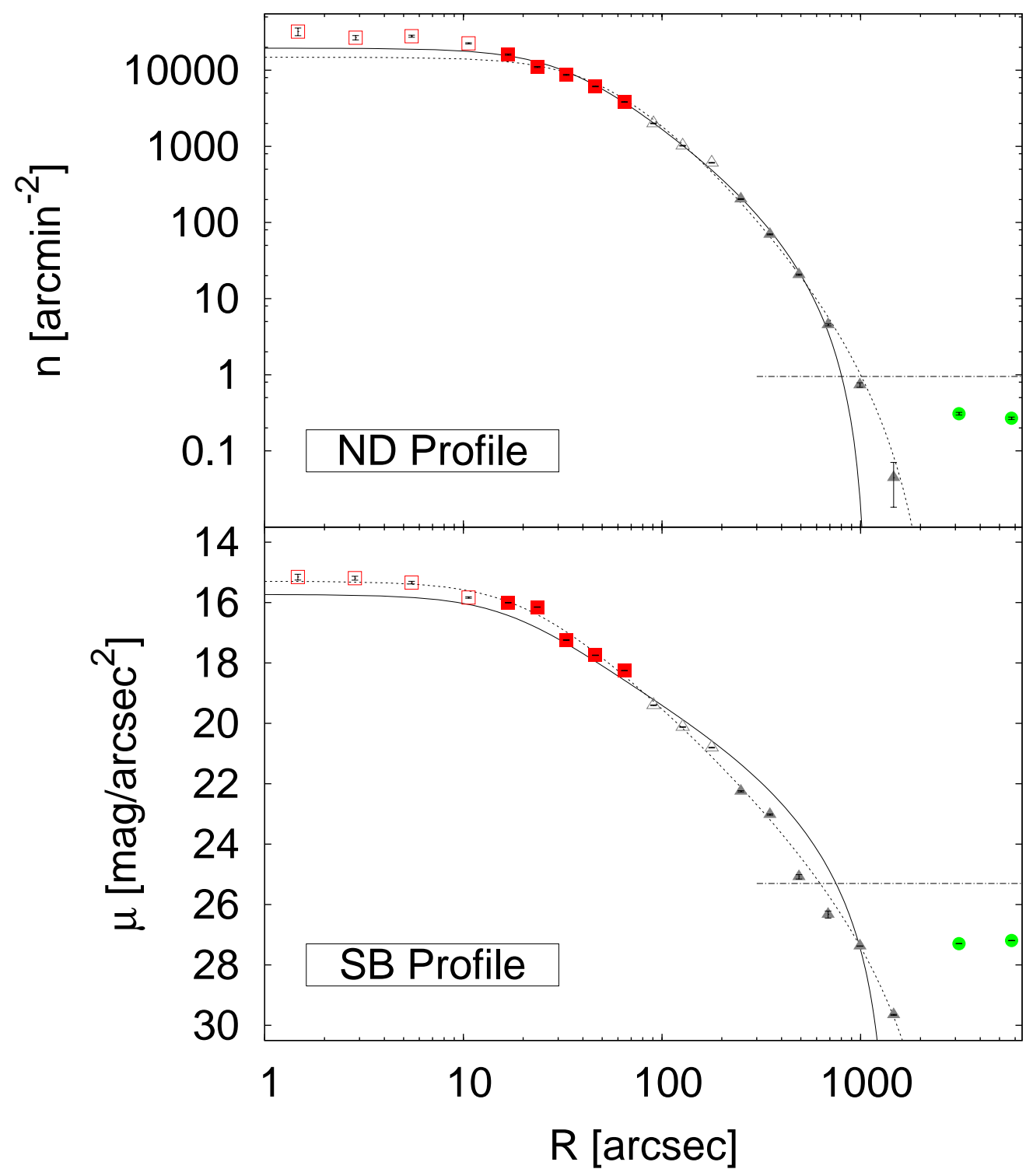

Fig. 8. - Fits by King and Wilson spherical models to the ND (top) and SB (bottom) profiles. Solid lines correspond to the King-model fits, dotted lines to Wilson-model fits; the horizontal dashed line shows the background level; errors are shown as vertical error bars. Red squares indicate data obtained from pointing $\gamma$, grey triangles those from the CFHT dataset, and green circles those from the SDSS. Empty symbols mark regions for which a completeness correction was applied. 


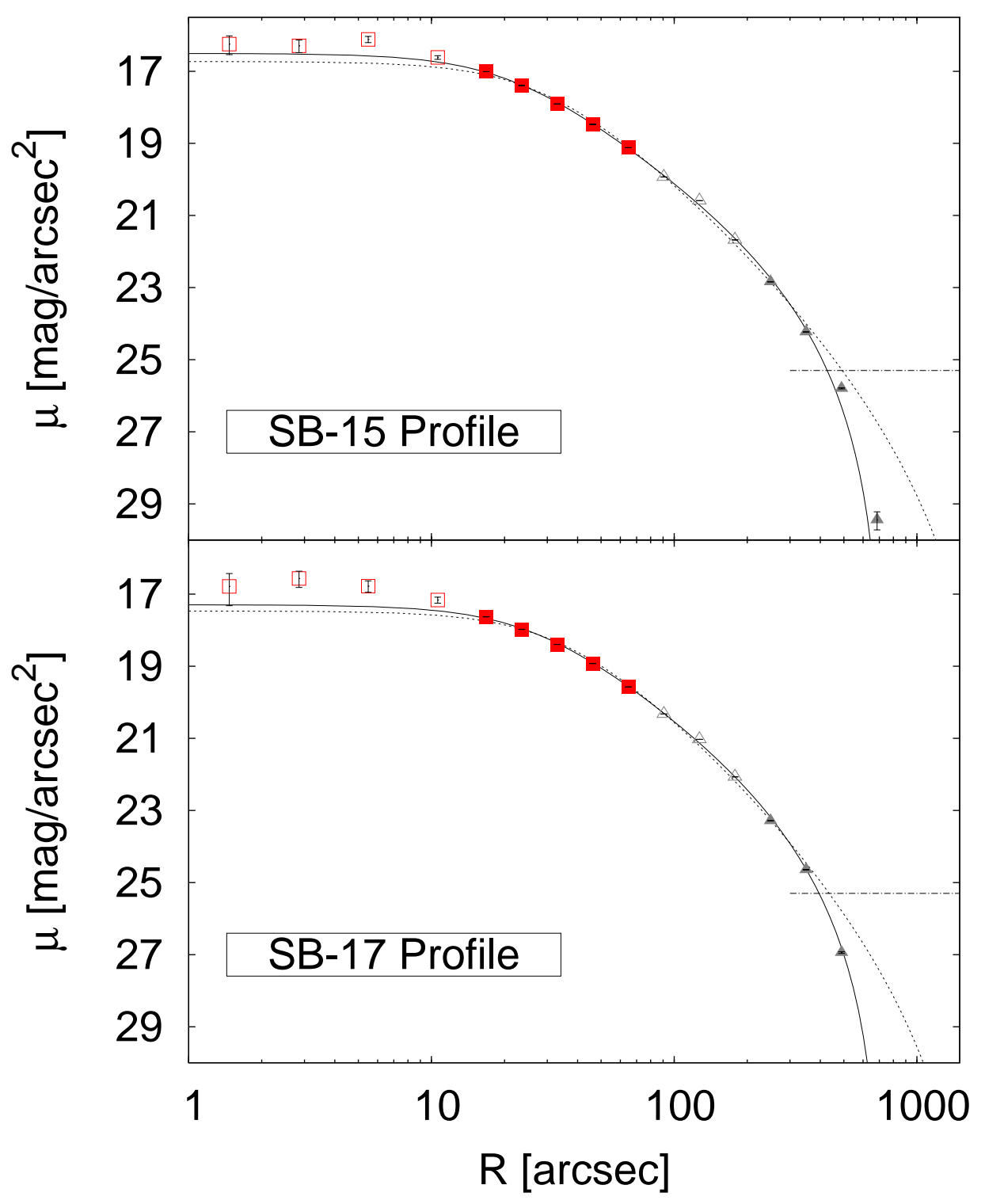

FIG. 9. - Fits by King and Wilson spherical models to the SB-15 (top) and the SB-17 (bottom) profiles. Solid lines correspond to the King-model fits, dotted lines to Wilson-model fits; the horizontal dashed line shows the background level. Data-points are indicated as in Fig. 8 and errors are shown as vertical error bars. 


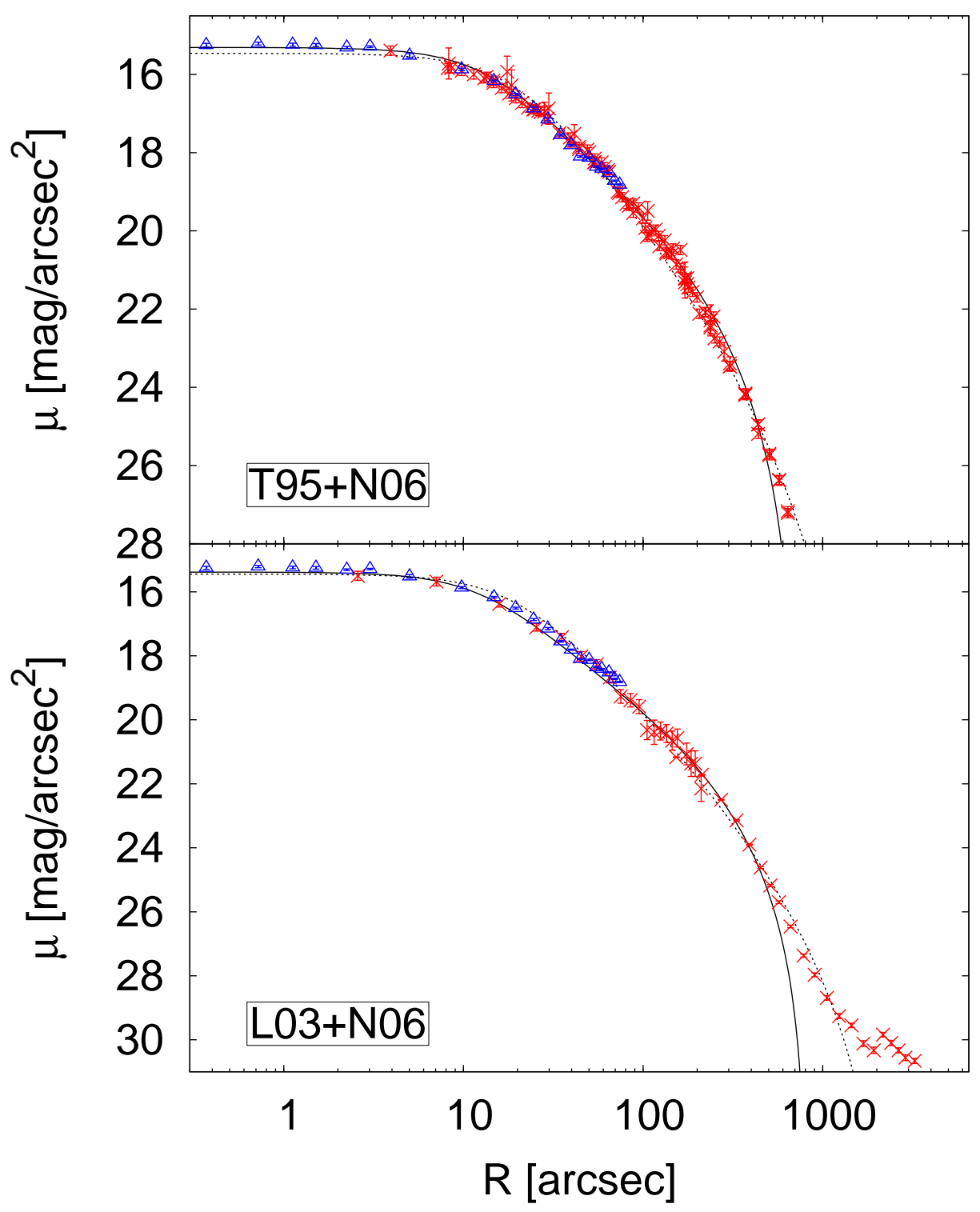

FIG. 10.- Fits by King and Wilson spherical models to the composite T95+N06 (top) and to the L03+N06 (bottom) profiles. Solid lines correspond to the King-model fits, dotted lines to Wilson-model fits; errors are shown as vertical error bars. Blue triangles indicate data from N06, red crosses those from other sources. 